\title{
Criminologie
}

\section{Le rôle de l'agent de probation avec une clientèle à incidence psychiatrique}

\section{Josette Leroux et Gaétan Larrivée}

Volume 21, numéro 2, 1988

Les soins de santé mentale en milieu carcéral

URI : https://id.erudit.org/iderudit/017266ar

DOI : https://doi.org/10.7202/017266ar

Aller au sommaire du numéro

Éditeur(s)

Les Presses de l'Université de Montréal

ISSN

0316-0041 (imprimé)

1492-1367 (numérique)

Découvrir la revue

Citer cet article

Leroux, J. \& Larrivée, G. (1988). Le rôle de l'agent de probation avec une clientèle à incidence psychiatrique. Criminologie, 21(2), 83-89.

https://doi.org/10.7202/017266ar
Résumé de l'article

The probation officer in a correctional milieu is confronted more and more by a clientele with various psycho-social pro b -lems, one of them psychiatric. By means of a case study, the authors examined the role of the probation officer in pre-sen-tence evaluation and post-sentence intervention vis-à-vis offenders and referrals to community resources

The first objective of the probation officer is to help in some way resolve the social conflict engendered by the offence and keep this in mind when dealing with the offender social services. 
LE RÔLE DE L'AGENT DE PROBATION AVEC UNE CLIENTÈLE À INCIDENCE PSYCHIATRIQUE

Josette Leroux* Gaétan Larrivée**

The probation officer in a correctional milieu is confronted more and more by a clientele with various psycho-social prob. lems, one of them psychiatric. By means of a case study, the authors examined the role of the probation officer in pre-sentence evaluation and post-sentence intervention vis-à-vis offenders and referrals to community resources

The first objective of the probation officer is to help in some way resolve the social conflict engendered by the offence and keep this in mind when dealing with the offender social services.

De façon générale, l'évolution de la psychiatrie moderne, de nouvelles approches d'intervention auprès des patients psychiatriques, des politiques sociales tenant compte des restrictions budgétaires ont favorisé l'utilisation du milieu ouvert comme terrain propice au traitement de la clientèle souffrant de troubles psychiatriques au détriment de l'institution traditionnelle. Pour une bonne partie de cette clientèle, surtout celle qui est supportée par un milieu familial et professionnel adéquat et qui accepte une médication et un traitement appropriés, ces nouvelles orientations semblent prometteuses. Toutefois, pour cette autre partie de la clientèle qui dispose de peu de ressources autant personnelles que sociales et qui s'insère mal dans le cadre des programmes de suivi en milieu ouvert, l'effet de la désinstitutionnalisation est tout autre. Pis encore, le client qui ne collabore pas avec les ressources en place et qui présente certains traits caractériels se retrouvera souvent seul. Rejeté socialement vu ses comportements aberrants, en quête de réponse à des besoins souvent fondamentaux et cela sans grand succès, cette portion de la clientèle psychiatrique en milieu ouvert présentera parfois des comportements sociaux inacceptables exigeant ainsi l'intervention policière et, par la suite, celle des tribunaux.

Dès l'entrée de ce type de contrevenant dans les réseaux judiciaires et correctionnels, l'agent de probation deviendra l'une des ressources les plus fréquemment interpellées tant par son rôle d'évaluateur au niveau présentenciel que dans ses rôles de surveillant et d'intervenant dans la gestion et l'application de la mesure sentencielle faisant appel au service de probation.

* Josette Leroux, agente de probation.

** Gaétan Larrivée, directeur du service de probation, Montréal Sud-Ouest, Service de probation, 3289, rue Saint-Jacques, Montréal (Québec) H4C IG8 
Quel est donc le rôle de l'agent de probation avec une clientèle à incidence psychiatrique? Comment pourrait-on définir sa contribution professionnelle? Afin de mieux illustrer l'apport de ce demier dans sa recherche ultime de résolution du conflit social engendrée par le délit, nous présenterons le cas de Monsieur $\mathrm{X}$ et la démarche suivie de l'évaluation initiale (rapport présentenciel) jusqu'à la fin de la période probatoire.

Monsieur X est âgé de trente-deux (32) ans et issu d'une fratrie composée de deux (2) filles et deux (2) garçons. Les parents sont toujours vivants et habitent une banlieue de la région métropolitaine. Le sujet vit encore avec eux et il n'a, à notre connaissance, jamais quitté le domicile familial.

Ce milieu familial présente des carences multiples. Nous relevons d'abord une relation très conflictuelle entre les parents et un déséquilibre au niveau des rôles joués par ces derniers auprès des enfants. Le père fut, en effet, très effacé, voire absent alors que la mère fut, de son côté, omniprésente au sein du foyer. Celle-ci fut par ailleurs hospitalisée à quelques reprises pour dépression nerveuse et nous décelons certains problèmes d'ordre psychiatrique chez deux enfants en plus du sujet. Un climat de paranoïa perdure également dans cette famille qui se sent continuellement menacée par le monde extérieur.

Au plan scolaire, Monsieur X a éprouvé des troubles d'apprentissage à partir du niveau primaire notamment à cause de problèmes de santé et il s'est difficilement rendu jusqu'en onzième année. Il a abandonné I'école à ce moment-là, soit à l'âge de dix-huit (18) ans, pour aller gagner sa vie. Il aurait, au total, travaillé huit (8) ou neuf (9) ans dont six (6) comme pressier dans une lithographie. Depuis trois (3) ans, il est tributaire de l'aide sociale et il semble de moins en moins apte à retourner sur le marché du travail.

Au plan criminologique, Monsieur X n'a pas manifesté de délinquance sévère durant sa jeunesse et ce n'est qu'à trente (30) ans qu'il connaît ses premiers démêlés judiciaires. Il plaide alors coupable à deux accusations de voies de fait, délits commis à très court intervalle et à l'endroit de deux (2) individus inconnus jusque-là du sujet. Dans les deux (2) cas, Monsieur X s'attaquera inopinément à ces individus parce qu'il les soupçonnait soit de lui avoir fait des avances, soit de l'avoir ridiculisé en le traitant d'homosexuel.

Une évaluation psychiatrique demandée par l'agent de probation dans le cadre du rapport présentenciel met en lumière les points sui- 
vants: malgré l'absence de thématique délirante structurée, l'expertise relèvera toutefois des préoccupations bizarres chez le sujet, un peu saugrenues et parfois paranoïdes vis-à-vis de l'entourage. On s'attaquera aussi à la dimension sexuelle du sujet, à sa gêne, sa difficulté de s'affirmer et de prendre ses distances face au milieu familial. En rapport avec ce dernier point, on notera chez le justiciable son manque d'autonomie, l'incapacité de s'assumer, une grande dépendance, son isolement social de plus en plus grand, beaucoup d'insécurité en même temps qu'un manque d'autocritique et une incapacité à évaluer ses problèmes, ses lacunes, même s'il peut les toucher du doigt superficiellement. Il est enfin recommandé que Monsieur $X$ bénéficie d'une supervision psychiatrique à l'intérieur d'une ordonnance de probation supervisée par un agent de probation.

Quant au rapport présentenciel, ce demier illustrera pour sa part les difficultés d'adaptation du sujet et le contexte familial perturbé (paranoïde) où il a évolué. Une problématique d'homosexualité refoulée et le besoin de prouver publiquement sa virilité apparaissent alors comme ayant été les éléments déclencheurs des passages à l'acte. Devant les problèmes d'identification et de personnalité perçus chez Monsieur X, il est également recommandé que ce dernier soit confié à la surveillance d'un agent de probation et qu'il se soumette à un traitement psychiatrique.

Suite à ces évaluations, le Tribunal opta pour une approche davantage curative et préventive. L'accusé fit alors l'objet d'une sentence suspendue d'une durée de deux (2) ans avec ordonnance de probation impliquant un suivi en probation et en psychiatrie.

L'agent de probation eut donc à assumer Monsieur $\mathrm{X}$ et avait la responsabilité de lui faire une offre légitimant son intervention professionnelle dans le cadre d'une mesure de probation et axée principalement sur la résolution du conflit social illustré par le délit.

Les coordonnées de notre intervention devaient donc considérer deux prérequis majeurs:

la perception du référant (Cour) et de la société de la nature du conflit reflété par le délit et son intérêt à recourir au programme de probation;

la perception qu'avait le contrevenant du délit imputé et l'intérêt qu'il avait à s'impliquer à la résolution du conflit.

Pour le référant, le délit était d'importance puisque dirigé contre la personne. Il apparaissait aussi évident qu'une problématique psychia- 
trique était à l'origine du geste délinquant et que cette problématique semblait s'actualiser de plus en plus en étant la source principale du conflit. Le référant notait également l'incompétence du milieu familial à assumer seul la prise en charge et l'absence de traitement spécialisé en psychiatrie vu le désintérêt du sujet pour ce faire. Compte tenu de ces faits, la Cour opta pour une mesure probatoire afin de s'asurer d'un contrôle minimal sur le contrevenant et d'apporter, au besoin, une aide psychosociale et psychiatrique au contrevenant.

Pour le contrevenant, le conflit était tout autre, il va de soi. Il n'assumait pas la responsabilité de son acte. Il se considérait davantage comme une victime qui s'était fait justice. Il n'était donc pas en conflit avec la société. De plus, il n'avait besoin d'aucun soin spécialisé, surtout psychiatrique car il n'était pas malade. Toutefois, étant donné sa crainte de la Cour, son seul intérêt à s'impliquer dans la résolution du conflit tel que déterminé par le référant (ordonnance de probation) était d'en respecter les conditions afin de s'éviter une nouvelle comparution. Il y avait donc une ouverture minimale.

Quant aux personnes intéressées à s'impliquer à la résolution du conflit (répondants sociaux), ils étaient peu nombreux vu l'isolement accru du justiciable. L'agent de probation et le psychiatre se disaient prêts à cheminer avec le client et la mère du sujet à collaborer vu son désir d'aider le justiciable à acquérir une meilleure autonomie.

L'offre de service, compte tenu des contraintes diverses et de la qualité de l'intérêt du justiciable, prit la forme suivante:

À court terme (l an)

- Donner au justiciable les moyens tangibles de s'éviter tout problème futur avec la justice. Comment :

- lui permettre de s'assurer du respect de ses conditions en lui présentant les ressources pertinentes (psychiatrie);

- lui offrir l'écoute, l'aide au besoin, et ainsi créer un lien avec l'agent de probation.

$\grave{A}$ moyen et long termes (1 an et + )

- Donner au justiciable les moyens lui permettant de diminuer sa souffrance (maladie, mésadaptation, isolement) et, ultimement, enrayer les causes de sa criminalité. Comment:

- lui démystifier la psychiatrie; 
- favoriser la création d'un lien davantage significatif avec le psychiatre, dépassant ainsi la simple obligation légale, et ce, dans le but de:

- découvrir son malaise;

- soulager le malaise;

- associer le milieu familial à cette démarche.

Dans les faits, la sentence s'est déroulée sans accrocs majeurs en ce sens que Monsieur X n'a pas récidivé et a fidèlement respecté son obligation de se rapporter au Service de probation. En outre, les entrevues constituèrent, pour le sujet, une occasion de verbaliser sur l'ensemble de ses préoccupations et lui permirent aussi d'entretenir une forme de contact, si précaire soit-il, en dehors du milieu familial. Il faut mentionner ici que, dans sa crainte d'être de nouveau «victimisé» par le système, le contrevenant décida d'éviter toute possibilité de confrontation avec autrui, en s'isolant de plus en plus chez lui, dans son monde. Par rapport à cela, l'approche de l'agent en fut une de dédramatisation par le reflet, notamment, d'éléments de réalité plus apaisants, non menaçants.

Du côté de la psychiatrie, il fut plus difficile d'amener le client à se conformer au plan initial, ce demier refusant obstinément de se reconnaître tout malaise de cet ordre. À ce chapitre bien précis, l'action de l'agent dut se situer à deux paliers, à savoir: celui du sujet et celui du psychiatre. Soulignons, à cette enseigne que Monsieur $X$ avait été évalué, au stade présentenciel, par le $\mathrm{D}^{\mathrm{r}} \mathrm{Y}$ et que ce dernier avait accepté d'assumer la supervision du patient durant la probation. Dès le début, cependant, un incident survint où Monsieur $X$ eut vraiment l'impression d'avoir été agressé par le médecin (il s'agissait d'une simple boutade) et suite à quoi Monsieur $\mathrm{X}$ refusa catégoriquement de poursuivre le traitement ave le $\mathrm{D}^{\mathrm{r}}$ Y. Peur réelle ou tentative de manipulation? Cela reste difficile à dire. Quoi qu'il en soit, le $\mathrm{D}^{\mathrm{r}} \mathrm{Y}$ révisa son diágnostic initial en statuant que le patient devenait franchement paranoïaque et qu'il devait absolument poursuivre un traitement. Il fut toutefois convenu que, compte tenu des difficultés du sujet avec les hommes, ce dernier pourrait bénéficier cliniquement, d'être référé à une femme. Ce qui fut fait.

Les échanges furent, de fait, plus faciles avec la nouvelle intervenante quoique le lien resta toujours relativement fragile. $\grave{A}$ un moment donné, l'agent de probation dut même négocier la poursuite du suivi avec la psychiatre, celle-ci considérant l'assiduité et la motivation du patient trop pauvres pour continuer. À ce stade, l'aspect légal dut aussi 
être utilisé pour reconvaincre le patient de ne pas abandonner le traitement. Suite à cela, ce dernier se montra un peu plus régulier vis-à-vis de la thérapie sans toutefois se reconnaître plus de besoins à ce niveau.

Ajoutons que la mère du sujet fut contactée à quelques reprises durant cette période et qu'elle fut un peu plus sensibilisée aux problèmes de son fils et au rôle qu'elle pouvait jouer auprès de lui. Elle s'est, en même temps, avérée une source d'information utile, notamment lorsque son fils traversait des moments plus difficiles.

En regardant, enfin, les résultats obtenus de cette démarche, on peut conclure qu'en dépit des changements mineurs perçus chez le contrevenant, le contexte légal dans lequel l'intervention s'est effectuée nous a, à tout le moins, permis de créer un lien entre Monsieur $\mathrm{X}$ et une ressource psychiatrique et nous permet d'espérer que le sujet aura recours à cette ressource s'il en venait à se désorganiser de nouveau.

L'exposé de ce cas type et les interventions de l'agent de probation permettent ici de mettre en lumière le rôle de ce dernier et surtout de légitimer son intervention professionnelle, peu importe la problématique présentée par le justiciable.

Partant du principe que tout individu appelé à comparaître devant les tribunaux suite à un délit se trouve nécessairement en conflit avec la société et qu'une sanction de cette société par l'entremise du tribunal se doit de lui être imposée afin de rétablir une certaine harmonie, plusieurs alternatives soit punitives, soit de réparation sociale, soit curatives ou préventives s'offrent au tribunal. L'un des principaux rôles de l'agent de probation sera donc d'expliquer la nature du conflit et de suggérer les meilleures avenues de solution au conflit tel qu'exprimé par le délit. Dans ce sens, toutes les sources possibles d'information lui seront nécessaires et il devra aussi aller chercher les expertises spécialisées, au besoin, afin d'étayer ses recommandations. Dans ce cas, le psychiatre et son expertise deviendront des éléments importants de compréhension quant à l'interprétation de la nature réelle du conflit.

D'autre part, un autre rôle de l'agent de probation dans le cadre d'un suivi probatoire sera de produire une offre de service au contrevenant respectant simultanément tant les intérêts du référant (Cour) que du contrevenant lui-même dans la recherche du retour d'une certaine harmonie de vie lui évitant ainsi les situations conflictuelles. Donc, après avoir évalué les intérêts de chacun et l'adhésion des parties à l'offre suggérée, tous les répondants sociaux identifiés, y incluant l'agent de probation, deviendront contributifs à l'atteinte du résultat 
recherché aussi limité soit-il. Ceci dit, partant du principe que le contrevenant en probation et/ou en libération conditionnelle demeure un citoyen à part entière et qu'il a droit à tous les services sociaux et de santé comme n'importe quel citoyen, toutes les ressources de notre société pourraient être mise à contribution. Dans le cas de Monsieur $\mathrm{X}$, il appert donc que des services psychiatriques aient été jugés prioritaires compte tenu de la détérioration constante du sujet.

Finalement et non le moindre, l'agent de probation aura pour rôle d'être le lien constant avec les ressources sociales mises à contribution afin de s'assurer du maintien de l'intérêt de part et d'autre à évoluer dans cette démarche correctionnelle. Pour ce faire, l'agent de probation devra avoir une bonne connaissance des ressources du milieu, être capable de présenter objectivement le contrevenant et situer l'aide recherchée, pouvoir définir la contribution de la ressource dans le cadre de la démarche correctionnelle et, au besoin, stimuler à nouveau l'intérêt des parties advenant des situations litigieuses. Comme on a pu le constater dans le cas présenté, l'agent de probation a dû faire appel à deux (2) ressources différentes et, à un certain moment, réactiver le processus.

Somme toute, l'agent de probation ne se substituera nullement à aucune autre ressource davantage spécialisée pour intervenir dans une problématique donnée. Au contraire, sa spécialité en fera davantage un intervenant dont l'action vise principalement à établir une démarche à l'intérieur du processus correctionnel visant ainsi le retour à une certaine harmonie sociale. Donc, ultimement, l'agent de probation sera le lien ou pivot privilégié entre le contrevenant, ses besoins et les systèmes judiciaires, correctionnels et communautaires. 